\title{
An Experimental and Theoretical Study of the Formation of Nanostructures of Self-Assembled Cyanuric Acid through Hydrogen Bond Networks on Graphite ${ }^{\dagger}$
}

\author{
Kavita Kannappan, Tova L. Werblowsky, Kwang T. Rim, Bruce J. Berne,* and \\ George W. Flynn* \\ Department of Chemistry and Nanoscale Science and Engineering Center (NSEC), Columbia University, \\ New York, New York 10027
}

Received: January 26, 2007; In Final Form: March 30, 2007

\begin{abstract}
The self-assembly of cyanuric acid into ordered nanostructures on a crystalline substrate, highly ordered pyrolytic graphite (HOPG), has been investigated at low temperature under ultrahigh vacuum (UHV) conditions by means of scanning tunneling microscopy in conjunction with theoretical simulations. Many domains with different self-assembly patterns were observed. One such domain represents the formation of an open 2D rosette (cyclic) structure from the self-assembly process, the first observation of this type of structure for pure cyanuric acid on a graphite substrate. Each self-assembled domain exhibits characteristic superstructures formed through different hydrogen bond networks at low coverage and low deposition rate. Experimental observation of coexistent, two-dimensional crystalline structures with distinct hydrogen bond patterns is supported by energy minimizations and molecular dynamics calculations, which show multiple stable structures for this molecule when self-assembled on graphite.
\end{abstract}

\section{Introduction}

Recent interest in nanometer size devices has encouraged the investigation of intra- and intermolecular forces that control and drive two-dimensional ordered nanostructures on a surface formed from the self-assembly of small molecular building blocks. A vast literature ${ }^{1-57}$ is available on the study of the selfassembly of various molecules on different substrates at liquid/ solid, ${ }^{1-14,17,22,23,25-27,29-34,36-38,42,44-49,54-57}$ gas (air)/solid, ${ }^{18}$ and vacuum/solid interfaces. ${ }^{15,16,19-21,24,28,35,39-41,43,50,51,53}$ The molecules studied vary from small cyclic ${ }^{10-12,44-46,50,51,54,56-58}$ to long-chain hydrocarbons with $22,23,25,26,29-34,36-38,42,44,45,47-49,55$ or without ${ }^{1-16}$ functional groups. In addition to the experimental studies, the literature abounds with examples of theoretical computational studies of self-assembled monolayers, often stemming from highly detailed surface science studies. These computational investigations also encompass a range of molecular systems that include functionalized alkanes, mono- and polycyclic rings, and molecules studied for their relevance to biological, materials/surface science, and nanoelectronics applications. ${ }^{59-74}$ These studies have revealed several ordered patterns from the self-assembly of a variety of molecules through the influence of different intermolecular forces that vary from strong hydrogen bond to weak van der Waals interactions between adsorbate species. Understanding the adsorbateadsorbate and adsorbate-substrate interactions that initiate and control self-assembly at interfaces has the potential to inform efforts to develop the next generation of novel materials to be used as optical and electrical devices, as well as chemical and biological sensors.

Recently, considerable progress has been made in synthesizing a new class of self-assembled molecules through noncovalent interactions involving hydrophobic, ionic interactions and

\footnotetext{
† Part of the special issue "Norman Sutin Festschrift".

* Authors to whom correspondence should be addressed. Phone: (212) 854-2186 (B.J.B.); (212) 854-4162 (G.W.F.).E-mail: bb8@columbia.edu (B.J.B.); gwf1@ @columbia.edu (G.W.F.).
}

hydrogen bonds. ${ }^{58,75-78}$ Self-assembly of small molecular building blocks through noncovalent interactions leads to the formation of unique structures that can be classified as supramolecular aggregates, fascinating assemblies observed in both nonbiological and biological chemical systems. These noncovalent interactions have been explored widely and found to lead to the formation of supramolecular crystals, ${ }^{16,18,20,24,28,35,39-41,43,46,50,54,56-58,75-85}$ assemblies that hold significant potential for the development of molecular devices. When the building blocks that make up these aggregates are biologically active molecules forming nanostructures on a surface, biocompatible materials and biosensors may be realized. If the building blocks have structural properties similar to biological molecules, they may find applications as medicinal agents. Understanding the structural complexity of biological macromolecules that derive much of their unique structure from noncovalent interactions leads inevitably to the study of model systems with similar structural properties.

Cyclic amides, in particular "cyanuric acid" (see Figure 1), which are structurally similar to amino acids, are promising candidates to use in the investigation of noncovalent interactions in the assembly and stabilization of biological systems on a surface. Cyanuric acid is a unique cyclic amide having three hydrogen bond donor and three acceptor sites, leading to a range of hydrogen bonding possibilities, both with other cyanuric acids as well as with polar solvents. A molecular model showing the tautomeric forms of cyanuric acid is given in Figure 1. Theoretical $^{86}$ and experimental ${ }^{87,88}$ evidence indicates that the molecule occurs in the most stable, keto form in all phases, gas, neutral solution, and sublimed film. In the keto form (b), arrows indicate six active sites for potential hydrogen bonding, three donor (in blue) and three acceptor (in red) sites that can be involved in noncovalent interactions with a maximum of six and a minimum of three other cyanuric acid molecules.

Cyanuric acid is known to form a linear structure in pure 3D crystals as well as when cocrystallized with solvent molecules. ${ }^{84}$ 


\section{Cyanuric Acid}

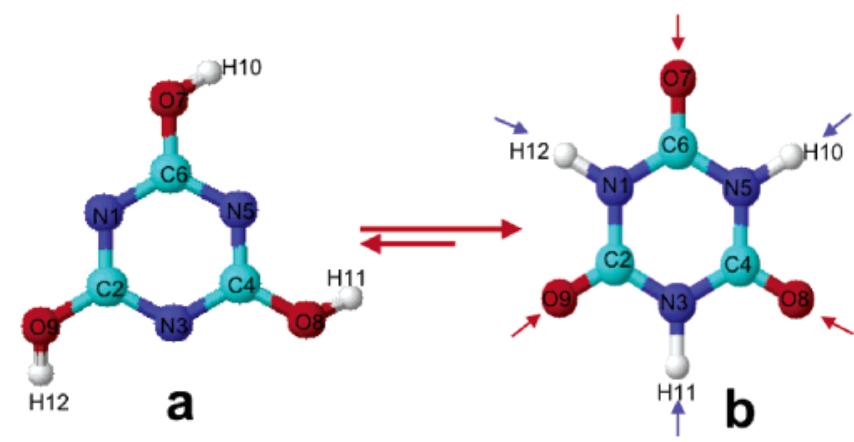

Figure 1. Molecular model of cyanuric acid showing different atoms marked in various colors. Cyanuric acid can occur in two tautomeric forms, enol (a) and keto (b). Both theory and experiments have shown that in all phases, gas, neutral solution, and sublimed films, the keto form is more stable than the enol form. The six arrows pointing toward the center of the molecule (b) mark the active sites for the formation of hydrogen bonds.

The 3D crystal structure of cyanuric acid retains this basic linear structure even though water solvent molecules are incorporated into the crystal lattice. $\mathrm{NMR}^{80,83}$ and X-ray crystallography ${ }^{82}$ studies have shown that the molecule forms a cyclic rosette structure only when crystallized with melamine in a 1:1 ratio.

In earlier studies at the air/solid interface, self-assembly of cyanuric acid on $\mathrm{Cu}(111)^{34}$ surfaces exhibited only one kind of linear monolayer formation, thought to be similar to that of the $3 \mathrm{D}$ crystal. A similar observation was made for one of the DNA bases, uracil, ${ }^{18}$ which was self-assembled on a $\mathrm{Cu}(111)$ surface and formed a similar close-packed 2D structure; uracil is the DNA base that most closely resembles cyanuric acid. It is interesting to note, however, that the sulfur derivative of this molecule, trithiocyanuric acid, in which all three oxygen atoms are replaced by sulfur atoms, forms both linear and rosette structures in pure $3 \mathrm{D}$ crystals. ${ }^{89}$

Different techniques including solid state NMR, ${ }^{83} \mathrm{X}$-ray crystallography, ${ }^{82,84,90-92}$ and hyper-Rayleigh scattering (HRS ${ }^{81}$ have been used to identify and understand the forces that control the fabrication of three-dimensional crystals as described above. However, in order to probe the atomic scale subtleties of hydrogen-bonded moieties of lower dimensionality, it is necessary to use scanning probe techniques. Scanning tunneling microscopy (STM) ${ }^{27}$ is one of the most recent and sophisticated techniques used to analyze two-dimensional structural properties. Its ability to provide detailed, high-resolution atomic and molecular information offers a unique opportunity to investigate the conformation and symmetry of the surface and of monolayer films on a substrate.

In the present investigation, 2D ordered nanostructures of cyanuric acid are imaged on graphite at the vacuum/solid interface using low-temperature STM. The experimentally observed images are compared to computationally modeled structures generated using combinations of geometry optimizations and molecular dynamics calculations.

\section{Experimental Section}

The experimental setup has been described in detail elsewhere. ${ }^{51,53}$ In brief, the STM experiments were carried out under ultrahigh vacuum (UHV) conditions inside a chamber evacuated continuously by an ion pump to a base pressure of $1 \times 10^{-10}$ Torr. The UHV chamber is equipped with a variable temperature scanning tunneling microscope (Omicron Vakuumphysik $\mathrm{GmbH}$ VT-STM) capable of scanning at temperatures ranging from
25 to $1500 \mathrm{~K}$. The chamber is also equipped with other surface analysis instrumentation, which includes a quadrupole mass spectrometer (Stanford Research Systems RGA 200), LEED (Princeton Research Instruments), and Auger (Staib Instruments, DESA 100). The substrate used in the present study was highly ordered pyrolytic graphite (HOPG, Advanced Ceramics, zyb grade). The HOPG was freshly cleaved and transferred immediately into the vacuum chamber through a turbo-pumped loadlock attached to the main chamber. STM tips were prepared by electrochemically etching a polycrystalline tungsten wire of diameter $0.25 \mathrm{~mm}$. Etched tips were annealed up to $\sim 800 \mathrm{~K}$ upon introduction into the UHV chamber. Prior to each experiment, the STM tip and the HOPG substrate were cleaned by heating to $\sim 750 \mathrm{~K}$ for $\sim 20 \mathrm{~min}$.

Monolayers of cyanuric acid were prepared by vacuum sublimation inside a small extension chamber attached to the loadlock. The molecules were vapor deposited using an evaporator with a molybdenum crucible and a type-K thermocouple. Cyanuric acid ( $98 \%$ pure) was obtained commercially from Aldrich and degassed at $350-360 \mathrm{~K}$ for $\sim 12 \mathrm{~h}$ prior to each deposition. The cyanuric acid sample was deposited onto the clean and warm $(330-335 \mathrm{~K})$ HOPG by heating the sample crucible to $415 \mathrm{~K}$. The molecules were deposited at a rate of $1.0-1.5 \AA / \mathrm{min}$ for $4.0-2.5 \mathrm{~min}$ to a total thickness of $\sim 4 \AA$. The rate of deposition was monitored with a quartz crystal microbalance (Sigma Instruments). Mass spectra of the deposited sample were recorded using the quadrupole mass spectrometer to make sure that the molecular monolayer formed on graphite is indeed that of cyanuric acid. The mass spectra obtained were comparable to those reported ${ }^{85}$ in the literature.

Following deposition of the cyanuric acid, the sample was cooled to approximately $80 \mathrm{~K}$, using a liquid helium flow cryostat, prior to scanning. In some cases the molecules were imaged at room temperature also, as specified in the figure captions. All images were acquired in constant current mode, and a real-time drift correction was applied to minimize the effects of thermal and mechanical drifts. The tunneling parameters for individual images are given in the figure captions, with the bias voltage polarity referenced to the sample.

\section{Theoretical Section}

The cyanuric acid molecule was modeled computationally using the program SIM, ${ }^{93}$ which incorporates the OPLS-AA force field. ${ }^{94}$ The OPLS potential function uses harmonic stretch and bend vibrational frequencies. In this case the partial charges were found to vary widely by force field, and thus, they were fit here using the JAGUAR ${ }^{95}$ program with a cc-pvtz-f basis set in a B3LYP functional with ESP charges constrained to fit the molecular quadrupole moment. The most appropriate intramolecular and Lennard-Jones parameters for this molecule in OPLS were found to be those adapted from the DNA base pair uracil, which has a very similar enol structure around the six-membered ring. Using the periodic cell information gleaned from the STM experiments described above as a basis for the two-dimensional structure in the initial calculations, energy minimization and molecular dynamics simulations were performed for small clusters of molecules adsorbed on a model graphite surface using SIM. Energy minimizations, using the truncated Newton algorithm ${ }^{96,97}$ were used to locate the minimum potential energy structure for each starting configuration. Constant NVT molecular dynamics (MD) simulations, thermally equilibrated with the Nose-Hoover chains method, ${ }^{98,99}$ were then performed on the minimized structures as described below. The MD simulations determine the effect of increasing 
temperature on a minimized low-energy structure and also allow an annealing of sorts to help locate the lowest-energy structures on a complicated energetic landscape.

The HOPG substrate is modeled using the Steele potential representation for a graphite surface. ${ }^{100}$ The top 2 graphite layer sheets are described using a fully corrugated potential, and an additional 38 layers below the top 2 sheets are modeled as a smoothed-out, attractive potential. This simplification of the graphite structure has proven to be more than adequate in other theoretical studies of surface adsorption. ${ }^{101}$

The adsorbate intramolecular and intermolecular energetics are described using Jorgensen's OPLS-AA force field. ${ }^{102}$ Intramolecular energy is described by harmonic bond stretches, bends, and torsions, as well as by Lennard-Jones (6-12) dispersions and repulsions, and by electrostatic interactions between atoms separated by at least three bonds (1-4 interactions), both of which are scaled by 0.5 in this model. Intermolecular energy is described by electrostatic interactions (point charges) and Lennard-Jones (6-12) van der Waals dispersion and repulsion interactions. The strength of the adsorbate-substrate interaction is given by the Steele energy term. Image charges between the molecules and the substrate can also be added to the model; however, they have been neglected in the present analysis because they are quite small and, when included, they do not alter the optimized structures.

\section{Results}

High-resolution images of cyanuric acid monolayers on graphite were obtained under identical (to within controllable error) ultrahigh vacuum and low deposition rate conditions, as described above in section II. A variety of monolayer structures were obtained, representative examples of which are shown in Figures 2-5; some of the contrasting structures were observed even during the same deposition experiment in different regions of the surface and represent coexisting structural domains. This suggests that the free energy of the different structures is similar to within $k T$, where $T$ is the evaporation/deposition temperature $(330-415 \mathrm{~K})$.

After close examination of the raw and computationally flattened images, the data were determined to consist of at least three distinct two-dimensional monolayer structures, with the rest of the scanned domains reflecting complex Moiré patterns superimposed on the spot patterns of the adsorbed molecules. This is a common phenomenon in STM experiments, where electronic effects are convoluted with structural and geometric positional information in the final images. Through careful examination and measurement of the distances and angles between observed bright or dark spots, however, a determination of actual molecular positions for the three clearly identified monolayer structures can be made and, further, the unit cell for each pattern can be used to eliminate any uncertainty in the observed 2D structures.

For each of the three different types of domains observed, measurements of intermolecular distances and angles were averaged both within the same image and over the total of all the images obtained for that monolayer structural type. An example of an image with a strong Moiré effect is shown in Figure $2 b$, where line profiles and other analyses show the adsorbed spot pattern to be contiguous throughout the dark and light regions; this type of image, though, was not used for quantitative analyses. The unit cell data for the types of structures represented in Figures 2a, 3, and 5 are tabulated in Table 1 . In each figure, the unit cell is represented by a rhombus

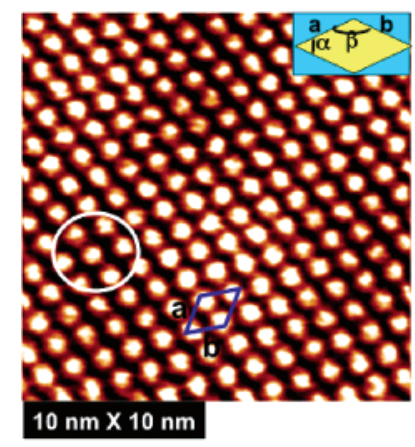

a

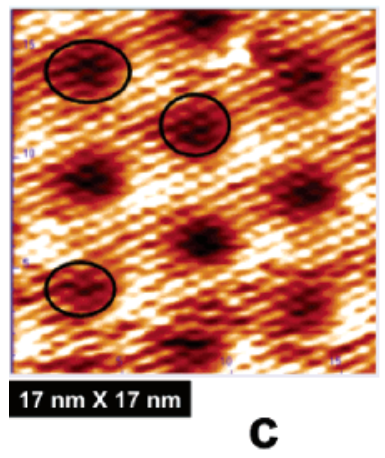

C

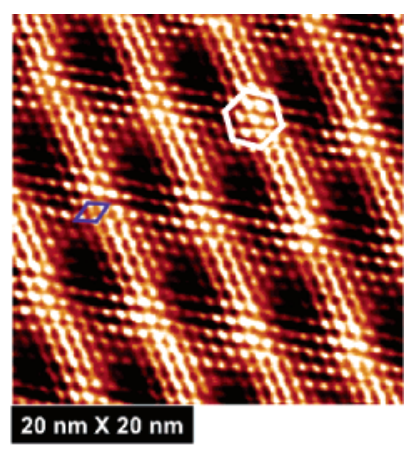

b

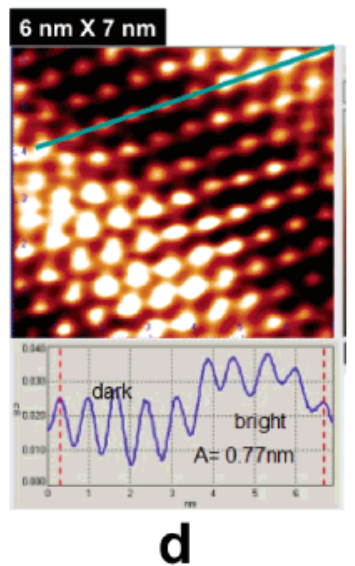

Figure 2. High-resolution STM images of monolayer structures of cyanuric acid having the same long-range order (acquired at $80 \mathrm{~K}$ with scanning conditions of $+1.9 \mathrm{~V}, 100 \mathrm{pA}$ ): (a) $10 \times 10 \mathrm{~nm}$; (b) $20 \times$ $20 \mathrm{~nm}$. Each bright spot represents a single cyanuric acid molecule. The basic repeat superstructure is marked by a white circle, which contains seven cyanuric acid molecules. The unit cell parameters (shown as a blue rhombus) and the nearest neighbor distances measured along the arrows (marked by a and $\mathrm{b}$ in part a) are tabulated in Table 1 . In part a, the yellow colored rhombus in the inset shows the angles $\alpha$ and $\beta$ of the unit cell. The images in parts $\mathrm{c}$ and $\mathrm{d}$ are examples of different scans of the heptamer structure that exhibited Moiré patterns. In part c, each spot inside the black circle represents cyanuric acid molecules in the dark region of the Moiré pattern similar to those observed in the bright region. Part $\mathrm{d}$ shows the zoomed-in image of a heptamer structure with a strong Moiré pattern where the spot pattern of the adsorbate layer is visible even in the dark regions. The line profile at the bottom of part $\mathrm{d}$, taken along the green line in the image, is mapped over dark to bright areas, showing that the intermolecular spacings do not change even though the electronic structure of the underlying graphite surface is modulating the intensity of the images.

and the regular repeat ("superstructure") patterns that will be discussed in the text below are shown using circular patches. The unit cells of all three film structures are rhomboid and characterized, respectively, by having one (heptamer superstructure), two (chicken wire superstructure), and six (flower superstructure) molecules per unit cell.

In addition to the well-resolved areas of the surfaces shown in Figures 2-5, some hazy-looking 2D gas-like (disordered) domains were also observed in scans of neighboring areas. These are indicative of the presence of very mobile monolayers that are most likely in the process of phase transition to more stable conformations. No clear, atomically resolved images of bare graphite, which might have provided information about the relative orientation between the adsorbed films and the graphite surface structure, were obtained in these experiments.

The monolayer structure shown in Figure 2 represents the simplest form of cyanuric acid self-assembly observed in the 


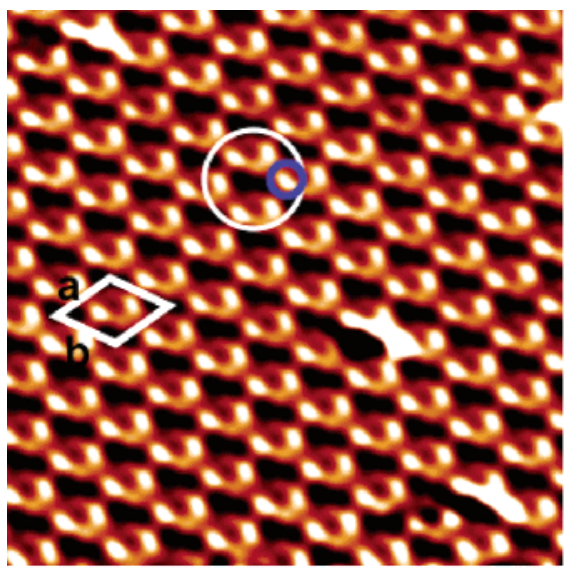

Figure 3. STM topograph of a cyanuric acid monolayer obtained at $80 \mathrm{~K}$ with the scanning parameters $+1.075 \mathrm{~V}$ and $132 \mathrm{pA}$. This image represents the chicken wire type of monolayer structure with a different long-range order from that of Figure 2. A single cyanuric acid molecule is represented by a small blue circle. The basic repeat hexamer superstructure in the monolayer is shown by a white circle containing six cyanuric acid molecules. The unit cell parameters (white rhombus) measured along the arrows $a$ and $b$ are listed in Table 1 .
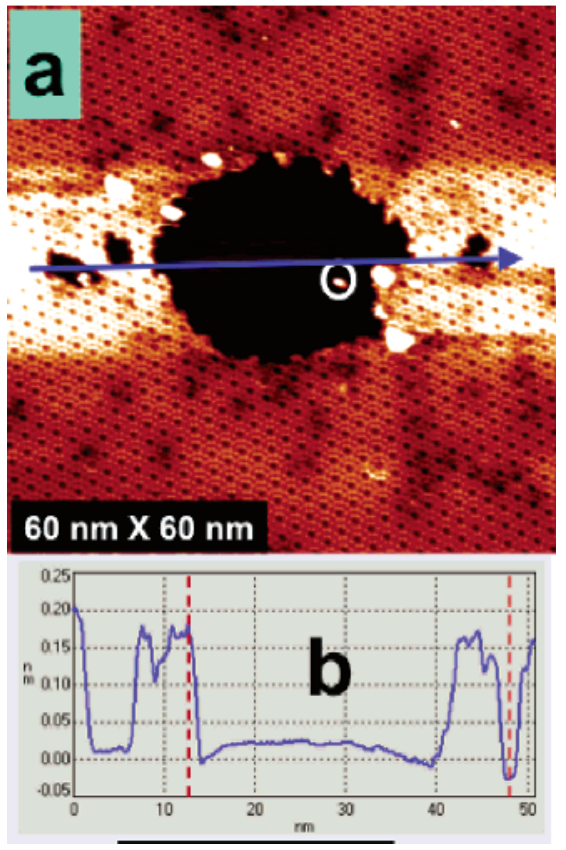

Figure 4. (a) STM image of a cyanuric acid monolayer obtained at $+1.9 \mathrm{~V}, 100 \mathrm{pA}$, and $80 \mathrm{~K}$. This corresponds to a third type of monolayer structure, the flower structure, observed in the cyanuric acid self-assembly on graphite under UHV conditions. The presence of an island of cyanuric acid molecules inside the topographical depression is shown in the white circle. (b) Representation of the line profile taken along the blue line in the STM image of part a. The depths along the line profile between the cyanuric acid monolayer and the three depressions were measured to be $0.17-0.21 \mathrm{~nm}$, the peak height generally observed for aromatic molecules laying flat on different substrates including graphite (see refs 88-90).

present study at both low $(80 \mathrm{~K})$ and room temperature (300 $\mathrm{K})$. It encloses one molecule in a regular hydrogen-bonded pattern with cell parameters $a=b$ (as tabulated in Table 1). On the basis of the above data and information about the length scales of an individual cyanuric acid molecule from calculations, each bright spot in the images is assigned to a single cyanuric acid molecule. This structure, which can be thought of as a sixmembered ring filled with a seventh cyanuric acid molecule (enclosed in the white circle in Figure 2a), will be referred to

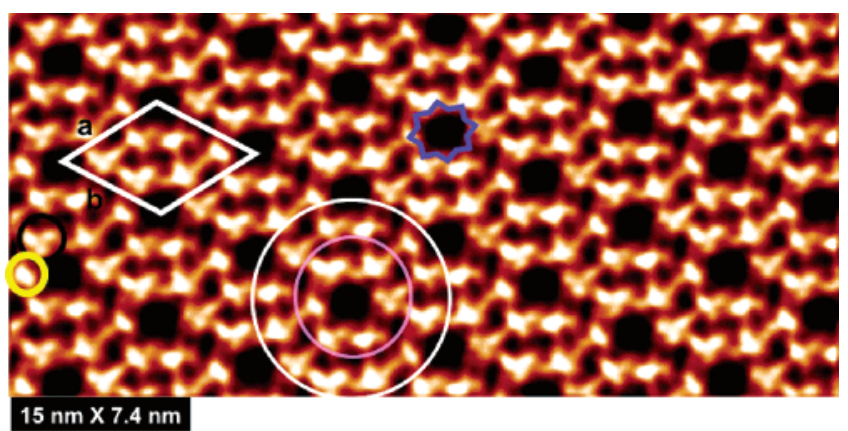

Figure 5. Submolecularly resolved STM topograph of a flower structure monolayer (shown in Figure 4a) obtained at $+1.9 \mathrm{~V}, 100 \mathrm{pA}$, and $80 \mathrm{~K}$. Concentric circles in white and purple containing 6 and 12 dots in the STM image (a) correspond to the 6 and 12 cyanuric acid molecules connected through hydrogen bonds. The small circles in black and yellow represent single cyanuric acid molecules with the one in the black circle more resolved than the one in the yellow circle. The cavity size shown as a blue star is measured to be $0.71 \times 0.76 \mathrm{~nm}$. The rhombus in white describes the unit cell. The nearest neighbor distances measured along the arrows $a$ and $b$ representing the unit cell in (a) are tabulated in Table 1

TABLE 1: Experimental and Theoretical Unit Cell Parameters of the Three Types of Monolayer Structures Shown in Figures 2-9

\begin{tabular}{lllrr}
\hline & \multicolumn{1}{c}{$a(\mathrm{~nm})$} & \multicolumn{1}{c}{$b(\mathrm{~nm})$} & \multicolumn{1}{c}{$\alpha$} & \multicolumn{1}{c}{$\beta$} \\
\hline heptamer (exptl) & $0.75 \pm 0.01$ & $0.75 \pm 0.01$ & $\sim 60$ & $\sim 120$ \\
heptamer (theor) & 0.68 & 0.68 & 60 & 120 \\
chicken wire (exptl) & $1.087 \pm 0.012$ & $1.087 \pm 0.013$ & $\sim 60$ & $\sim 120$ \\
chicken wire (theor) & 1.08 & 1.08 & 60 & 120 \\
flower (exptl) & $1.96 \pm 0.03$ & $1.84 \pm 0.01$ & $\sim 60$ & $\sim 120$ \\
flower (theor) & 1.80 & 1.80 & 60 & 120
\end{tabular}

as the "heptamer" in this work. The regular $60^{\circ}$ unit cell pattern clearly seen in the images shows this to be a simple onemolecule unit cell; however, the large error bars are due to the measurement of spots reflecting electron density in combination with Moiré effects from the underlying graphite surface (shown in Figure 2b). These effects are responsible for the relatively wide range of angles and distances seen from image to image even where the same structure is definitively observed visually.

The STM image shown in Figure 3 corresponds to another domain with a different type of molecular arrangement. This type of open structure where the molecules arrange in regular six-membered rings (denoted a "chicken wire" structure in previous work) has been observed in other hydrogen-bonded ring systems. ${ }^{56,103}$ The unit cell is marked by a white rhombus, and the cell parameters obtained are tabulated in Table 1 . The spacing of the hole in the center of each of the chicken wire rings is measured to be $\sim 1 \mathrm{~nm}$ in width.

Figures 4 and 5 represent the third type of monolayer structure that was detected on multiple occasions. In Figure 4, the longrange order of this domain is evident, and a wide unoccupied graphite region can be seen surrounded by the adsorbate monolayer. Inside the bare graphite space, a circle marks a clump of cyanuric acid molecules. The line profile shown in Figure $4 \mathrm{~b}$ is taken along the blue line in Figure $4 \mathrm{a}$ and gives evidence that the dark section is in fact bare graphite. Figure 5 shows the double-ring structure that we will refer to as the "flower" arrangement in this work. The unit cell is represented by a white rhombus, and averaged cell parameters measured from many different images of the same structure are given in Table 1.

\section{Theoretical Results}

A set of simulations was performed to examine the stability and mobility of the observed STM structures and to quantify 


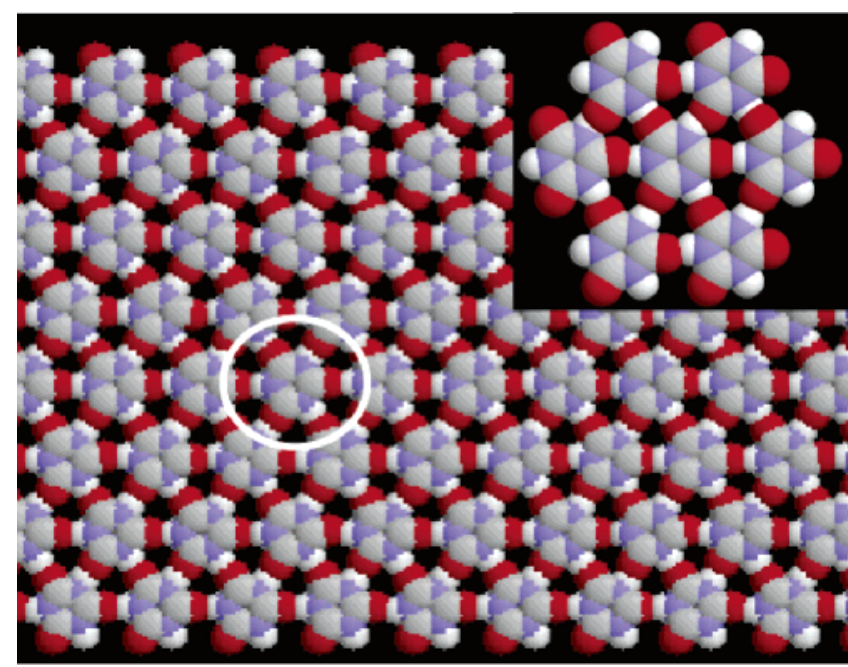

Figure 6. Patch of cyanuric acid molecules representing the configuration of the lowest-energy simulation of the heptamer structure, corresponding to the experimentally observed image shown in Figure 2 . The inset shows an enlarged version of the basic seven-molecule heptamer structure. Red = oxygen; white = hydrogen; gray = carbon; blue $=$ nitrogen

the energetics of each type of monolayer. To this end, a nonperiodic cluster of cyanuric acid molecules was first built for each of the heptamer, chicken wire, and flower types of monolayer that best approximated the experimental distances and angles. These initial structures were then energy minimized, and the resulting lowest-energy structures for each arrangement were propagated at the imaging temperature $(80 \mathrm{~K})$. In addition, a set of simulations was performed in which the minimized monolayers were annealed to $300 \mathrm{~K}$ and cooled slowly to 80 $\mathrm{K}$. This ensures that the structures used as templates for building the larger periodic monolayers were indeed the lowest-energy units, not just species energetically trapped at the reduced simulation temperature. These smaller templates were then extracted from the stable, internal portions of the larger clusters after long times and replicated in two dimensions to produce large periodically bound adsorbate layers. These structures were then again minimized and propagated using molecular dynamics at the experimental deposition and scanning temperatures, but now the appropriate boundary condition was applied in each case. Finally, a comparison of the energetics of the final three different structures was made, using the bulk properties of the periodically replicated $2 \mathrm{D}$ cells to analyze the differences in energy of the competing structures on a per molecule basis. The final structure for each type of observed monolayer is shown in Figures 6-8. The parameters of the unit cells for each resulting polymorph match very well with experiment, as can be seen in Table 1 .

\section{Discussion}

A. The Importance of Hydrogen Bonding. As shown in Figures $2-5$, the self-assembly of cyanuric acid on graphite leads to multiple monolayer structures. The formation of many domains with distinct monolayer structure arises from different hydrogen bond patterns that hold the cyanuric acid molecules together (similar to the case of the DNA base adenine, ${ }^{28}$ which forms two different monolayer structures through two distinct types of hydrogen bond patterns). For each of the structures imaged, the corresponding simulated structure is shown (in Figures 6-8), with a magnified view to enable the exact bonding for each configuration to be analyzed.

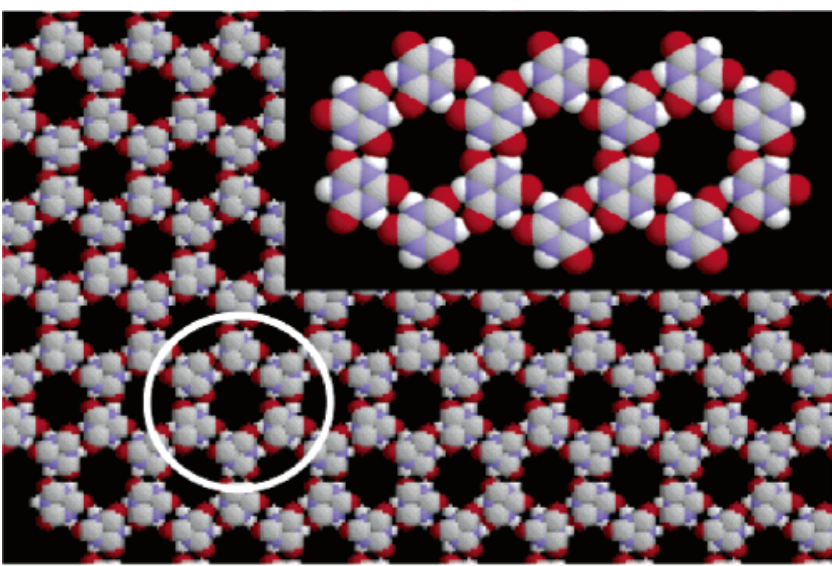

Figure 7. Large simulated patch of the chicken wire structure corresponding to the experimentally observed image in Figure 3. The dark holes in the centers of the six-membered rings are measured to be $\sim 1 \mathrm{~nm}$. The inset shows an enlarged image of three rings of the chicken wire structure. The atom color scheme is the same as that of Figure 6.

The monolayer shown in Figure 2a,b, with its theoretically simulated counterparts in Figure 6, corresponds to the simplest form of monolayer observed in these STM experiments with a $60^{\circ}$, one molecule per unit cell, offset structure. This heptamer structure is in fact similar to that seen in STM images of bare HOPG; however, the unit cell parameters as measured are markedly different from those of bare graphite. That, in addition to the height information for the dosed surface and the individual defects in the monolayer, inspires confidence that the image represents individual cyanuric acid molecules.

The cyanuric acid molecules in Figure 2 form a network of "super hydrogen bonds", as shown in Figure 9a. Electrostatic interactions among these molecules place them in a cooperatively strengthened formation where three molecules (or equivalently six atoms) share the hydrogen bond charge density. This structure was observed most often in the experimental data and is the most close-packed of all the repeat patterns imaged.

In marked contrast to the tight heptamer structure described above, the other two types of adsorbate arrays exhibit cyanuric acid molecule structures held together by hydrogen bonds more typical of carboxylic acid functionalities. Open cyclic (chicken wire, Figure 3) and double-ringed (flower, Figures 4 and 5) structures are readily observed. (The monolayer structures shown in Figures $4 \mathrm{a}$ and 5 are the same; Figure 5 is the enlarged image of Figure 4a.) The theoretically simulated chicken wire and flower structures are shown in Figures 7 and 8, and they demonstrate the different possible combinations of hydrogen bonds available to these molecules. The chicken wire structure forms only the typical hydrogen-bonded dimer (Figure 9b), while the flower structure combines the type of bonds formed in the heptamer (Figure 9a and inset of Figure 6) with those of the chicken wire arrangement shown in Figure 9b.

The three coexistent structures mentioned above are examined and compared energetically in Table 2. These energies are normalized by the number of molecules used in the simulation and compared in the last column to the energy of a single isolated molecule adsorbed on a graphite surface. One isolated molecule has energy associated with it due to the internal strain inherent in conjugated rings and the close proximities of atoms to each other due to the geometric constraints. In addition, electrostatic and Lennard-Jones energies (van der Waals type repulsions and dispersions) are calculated for atoms that are within the same molecule but separated by more than three bonds. 


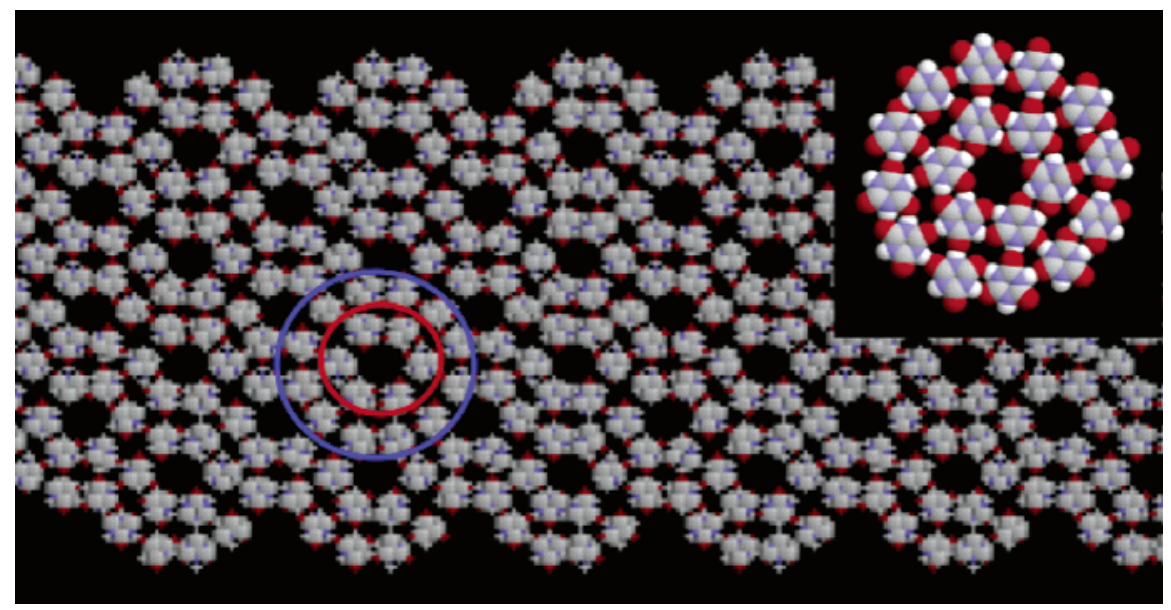

Figure 8. Simulation of the flower or rosette structure. The two concentric circles correspond to those observed experimentally and shown in Figure 5, with the smaller ring enclosing 6 molecules in a chicken-wire-like structure and the larger ring incorporating 12 cyanuric acids. The inset shows an enlarged image of just 12 molecules of the flower structure. The internal hole is $\sim 1 \mathrm{~nm}$ in diameter, and the atom color scheme is the same as that of Figures 6 and 7.
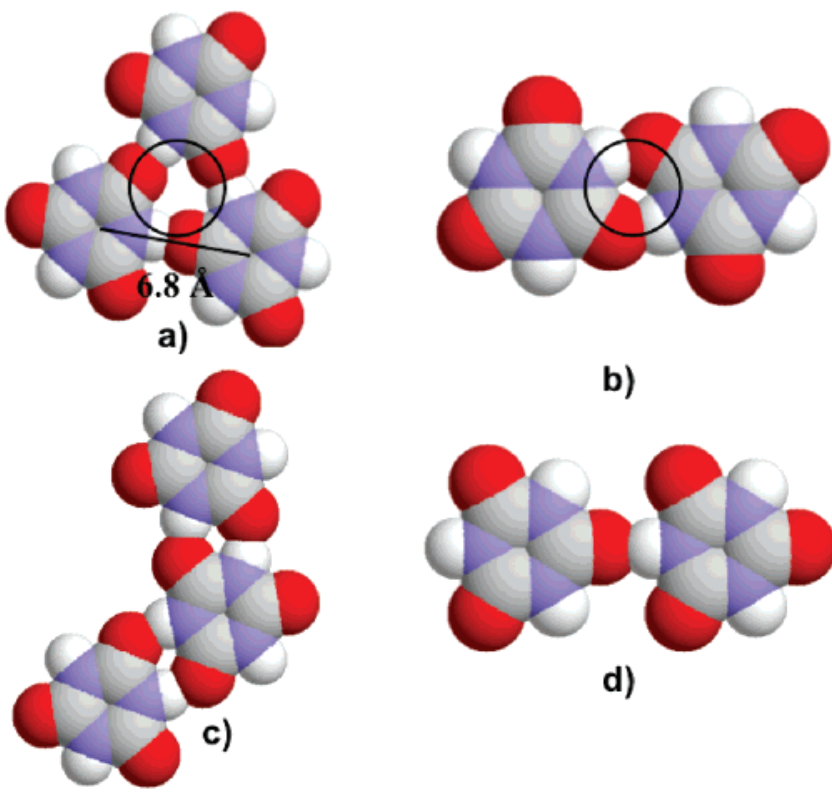

b)

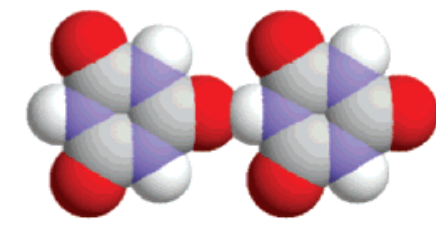

d)

Figure 9. (a) The basic unit of the super hydrogen-bonded trimer observed in both experiment and simulation for the heptamer and flower structures. This cluster was used to calculate the energy listed in column 1 of Table 4. (b) The classic dimer double hydrogen bond observed in both experiment and simulation for the chicken wire and flower structures. (c) A three-molecule cluster of the type observed in experiments and simulations for the chicken wire structure. (d) A classic single hydrogen bond of the type observed in the flower structure connecting the inner 6-membered ring to the outer 12-membered ring (see inset of Figure 8). The atomic color scheme is the same as that of Figures 6-8.

The results of these calculations clearly show that the heptamer structure is energetically the most favorable overall. The flower structure does slightly better in the category of Lennard-Jones interactions, but the electrostatic interactions clearly tilt the balance in favor of the "super" hydrogen bond formation of the heptamer. It is important to note, however, that although the chicken wire structure is less energetically favored compared to the other two structures, it is still significantly stabilized relative to an isolated adsorbed molecule. Hydrogen bond formation (Figure 9a,b) is clearly an energylowering event in the monolayer adsorption. Nevertheless, this detailed analysis begs the question of why the chicken wire type

TABLE 2: Energies for the Different Monolayer Structures Observed in the STM Experiments Compared to the Energy for One Isolated, Adsorbed Cyanuric Acid Molecule

\begin{tabular}{lcccc}
\hline & \multicolumn{3}{c}{ chicken } & one \\
& weptamer & wire & flower & isolated \\
\hline total potential energy $^{a}$ & -98.47 & -89.93 & -95.85 & -78.63 \\
electrostatic $^{b}$ & -90.98 & -84.77 & -86.24 & -71.69 \\
Lennard-Jones $^{c}$ & 4.774 & 7.029 & 4.446 & 7.640
\end{tabular}

${ }^{a}$ Energies in $\mathrm{kcal} / \mathrm{mol}$ are reported on a per molecule basis. ${ }^{b}$ The electrostatic energy refers to the sum of the pairwise interactions of the partial charges assigned to the atoms using the force field as defined in the text (see Theoretical Section). ${ }^{c}$ The Lennard-Jones energy refers to the nonbonded van der Waals interactions between two atoms at short range and takes the form of a standard 6-12 potential for repulsions and dispersions, respectively (see Theoretical Section).

TABLE 3: Comparison of Energies for the Structures Shown in Figure 10

\begin{tabular}{|c|c|c|c|c|}
\hline & $\begin{array}{c}\text { heptamer, } \\
\text { three } \\
\text { molecules }\end{array}$ & $\begin{array}{c}\text { heptamer, } \\
\text { two } \\
\text { molecules }\end{array}$ & $\begin{array}{l}\text { chicken } \\
\text { wire, three } \\
\text { molecules }\end{array}$ & $\begin{array}{c}\text { chicken } \\
\text { wire, two } \\
\text { molecules }\end{array}$ \\
\hline total potential energy ${ }^{a}$ & -87.64 & -79.50 & -82.12 & -81.62 \\
\hline electrostatic ${ }^{b}$ & -78.71 & -74.65 & -79.01 & -77.04 \\
\hline Lennard-Jones ${ }^{c}$ & 7.393 & 7.631 & 7.406 & 7.735 \\
\hline
\end{tabular}

${ }^{a}$ Energies in $\mathrm{kcal} / \mathrm{mol}$ are reported on a per molecule basis. ${ }^{b}$ The electrostatic energy refers to the sum of the pairwise interactions of the partial charges assigned to the atoms using the force field as defined in the text (see Theoretical Section). ${ }^{c}$ The Lennard-Jones energy refers to the nonbonded van der Waals interactions between two atoms at short range and takes the form of a standard 6-12 potential for repulsions and dispersions, respectively (see Theoretical Section).

of bonding is observed at all. To decipher this puzzle, a closer look at the bonds is needed.

An analysis of the basic structural units of the heptamer versus the chicken wire structures shows that the former is composed of a three-molecule basic unit (Figure 9a), whereas the latter has an interaction that is completely satisfied with a twomolecule bond (Figure 9b). To understand this quantitatively, the energies of three molecules of each type of cluster (again reported per molecule) were compared to the energies of only a pair of molecules in both configurations. As expected and reported in Table 3, the heptamer structure is energetically favored over the chicken wire structure as long as three molecules (and therefore the complete three-molecule hydrogen bond) are considered (Figure 9a,c). However, a calculation of 


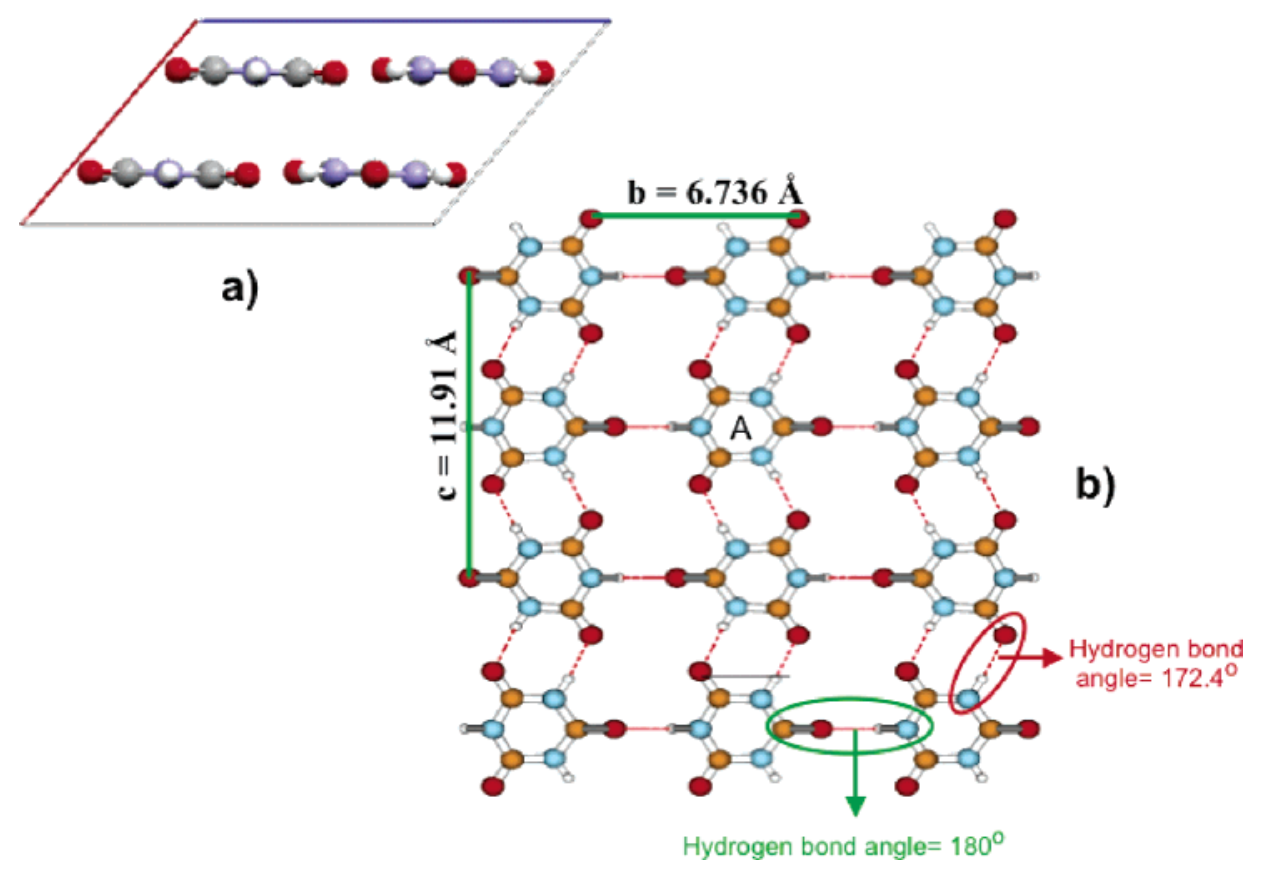

Figure 10. (a) Representation of a side view of the 3D unit cell of the cyanuric acid crystal structure. (b) Two-dimensional cut along the $b$ and $c$ crystallographic axes of a crystal of cyanuric acid containing 12 molecules. A single cyanuric acid molecule, labeled "A", is surrounded by eight other cyanuric acids in a plane. Only four of these are chemically bonded to A by two different hydrogen bond patterns (see refs $90-92$ ). The cell parameters along $b$ and $c$ are marked by green lines and measured to be 6.736 and $11.91 \AA$, respectively (see ref 90). The two types of hydrogen bonds observed in the crystal structure are marked by red and green ovals.

only the dimer energy of each type clearly favors the chicken wire structure, which has a total of four atoms involved in a cooperatively strengthened hydrogen bond. In contrast, the heptamer linear dimer has only two atoms joined along a line at an angle of $180^{\circ}$. This suggests that the monolayer structures seen on graphite are to some degree kinetically trapped into metastable configurations. This is borne out by calculations in which randomly oriented molecules at high temperatures were allowed to adsorb onto graphite without boundary conditions or initial structural bias. ${ }^{104}$ Even after very long simulation times, all of the structures mentioned above are seen, and when the molecules adsorb in a pairwise fashion onto the graphite after collisions in the gas phase, they come down in the chicken wire dimer configuration.

B. Comparison of 2D Self-Assembly and the 3D Crystal Structure of Cyanuric Acid. In addition to the structural information gleaned from the scanning tunneling microscopy experiments, there is an obvious advantage in obtaining 3D crystal structure information for comparison to 2D crystal-like patterns as imaged in STM experiments. The 3D crystal structure of cyanuric acid has been well studied. ${ }^{90-92}$ The unit cell parameters from X-ray crystallographic data are $a=7.749 \AA$, $b=6.736 \AA, c=11.912 \AA, \alpha=90^{\circ}, \beta=130.69^{\circ}$, and $\gamma=$ $90^{\circ}$. Part a of Figure 10 shows a side view of the 3D unit cell, and part b shows a $2 \mathrm{D}$ cut through this $3 \mathrm{D}$ crystal structure along the $b$ and $c$ axes.

The planar crystal structure has elements of both the heptamer structure (a linear hydrogen bond) and the chicken wire structure (the $\mathbf{c}$ vector direction exhibits a classic dimer hydrogen bond). To understand why this type of combination structure is never seen in two-dimensional monolayer experiments, two calculations of the crystal structure were performed. In the first, a onelayer cut of the crystal structure was replicated periodically in 2D on graphite, and in the second, another crystalline layer was added. The results are tabulated in Table 4 and clearly show that the energetic advantage gained from the 3D crystal type of structure is only observed when a second solid-like layer is
TABLE 4: Comparison of Energies for One-Layer and Two-Layer Periodically Bound Crystal Structures for Cyanuric Acid Molecules

\begin{tabular}{lcc}
\hline & $\begin{array}{c}\text { one layer of } \\
\text { crystal structure }\end{array}$ & $\begin{array}{c}\text { two layers of } \\
\text { crystal structure }\end{array}$ \\
\hline total potential energy $^{a}$ & -88.095 & -91.8799 \\
electrostatic $^{b}$ & -93.00977 & -93.7748 \\
Lennard-Jones $^{c}$ & 8.610365 & 3.91198
\end{tabular}

${ }^{a}$ Energies in $\mathrm{kcal} / \mathrm{mol}$ are reported on a per molecule basis. ${ }^{b}$ The electrostatic energy refers to the sum of the pairwise interactions of the partial charges assigned to the atoms using the force field as defined in the text (see Theoretical Section). ${ }^{c}$ The Lennard-Jones energy refers to the nonbonded van der Waals interactions between two atoms at short range and takes the form of a standard 6-12 potential for repulsions and dispersions, respectively (see Theoretical Section).

formed above the first on the graphite substrate. Otherwise, the stabilization gained from the attractive electrostatic energy is more than cancelled out by the repulsive Lennard-Jones energies. The second offset graphite-like layer is crucial in understanding the relative stabilities of the $3 \mathrm{D}$ versus $2 \mathrm{D}$ structures of cyanuric acid. This provides additional support for the idea that, in ambient liquid-solid experiments on graphite, only the first layer that is in direct contact with graphite has any significant crystalline structure.

\section{Summary and Conclusions}

Using low-temperature scanning tunneling microscopy under ultrahigh vacuum conditions in conjunction with theoretical simulations, the self-assembly of cyanuric acid on HOPG was investigated. Due to the presence of six active hydrogen bond sites in the molecule, self-assembly leads to multiple structures which can be categorized according to the types of hydrogen bonding between the molecules. One is a close-packed honeycomb structure, and the other two well-resolved structures exhibit different repeat patterns involving six-membered rings with nanometer-sized holes in their centers. The multiple 
coexistent structures are explained as local minima in a complex free energy landscape where the different opportunities available for the hydrogen-bonded moieties trap the adsorbing molecules into starkly contrasting longer-range structures. Cyanuric acid is unique in forming many monolayer structures due to distinct hydrogen bond patterns involved in the self-assembly. Different initial nucleation processes drive the self-assembly to form different monolayers under similar experimental conditions.

Acknowledgment. This work was supported by grants from the National Science Foundation (CHE-03-52582 to G.W.F. and CHE-03-16896 to B.J.B.). Partial support was provided by the NSEC Program of the National Science Foundation under Award No. CHE-01-17752 and by the New York State Office of Technology, Science, and Academic Research (NYSTAR).

\section{References and Notes}

(1) Groszek, A. J. Proc. R. Soc. London 1970, 314, 473.

(2) Findenegg, G. H. J. Chem. Soc., Faraday Trans. 1973, 68, 1799.

(3) Findenegg, G. H. J. Chem. Soc., Faraday Trans. 1973, 69, 1069.

(4) McGonigal, G. C.; Bernhardt, R. H.; Thomson, D. J. Appl. Phys. Lett. 1990, 57, 28 .

(5) McGonigal, G. C.; Bernhardt, R. H.; Yeo, Y. H.; Thomson, D. J. J. Vac. Sci. Technol., B 1991, 9, 1107.

(6) Buchholz, S.; Rabe, J. P. J. Vac. Sci. Technol., B 1991, 9, 1126.

(7) Allen, M. J.; Balooch, M.; Subbiah, S.; Tench, R. J.; Siekhaus, W.; Balhorn, R. Scanning Microsc. 1991, 5, 625.

(8) Heckl, W. M.; Smith, D. P. E.; Binnig, G.; Agges, H. K. J.; Hansch, T. W.; Maddocks, J. Proc. Natl. Acad. Sci. U.S.A. 1991, 88, 8003.

(9) Liang, W.; Whangbo, M.-H.; Wawkuschewski, A.; Cantow, H. J.; Magoonov, S. N. Adv. Mater. 1993, 5, 817.

(10) Wawkuschewski, A.; Cantow, H. J.; Magonov, S. N.; Liang, W.; Whangbo, M.-H. Adv. Mater. 1993, 5, 821.

(11) Wawkuschewski, A.; Cantow, H. J.; Magonov, S. N. Langmuir 1993, 9, 2778.

(12) Magonov, S. N.; Wawkuschewski, A.; Cantow, H. J.; Liang, W.; Whangbo, M.-H. Appl. Phys. 1994, 59, 119.

(13) Cyr, D. M.; Venkataraman, B.; Flynn, G. W. Chem. Mater. 1996, 8,1600 .

(14) Cyr, D. M.; Venkataraman, B.; Flynn, G. W.; Black, A.; Whitesides, G. J. Phys. Chem. 1996, 100, 13747 .

(15) Strohmaier, R.; Ludwig, C.; Petersen, J.; Gompf, B.; Eisenmenger, W. Surf. Sci. 1996, 351, 292.

(16) Nakagawa, T.; Tanaka, H.; Kawai, T. Surf. Sci. 1997, 370, L144. (17) Claypool, C. L.; Faglioni, F.; Goddard, W. A., III; Gray, H. B.; Lewis, N. S.; Marcus, R. A. J. Phys. Chem. B 1997, 101, 5978. 85

(18) Sowerby, S. J.; Peterson, G. B. J. Electroanal. Chem. 1997, 433,

(19) Lee, H. S.; Iyengar, S.; Musselman, I. H. Langmuir 1998, 14, 7475. 376.

(21) Strohmaier, R.; Petersen, J.; Gompf, B.; Eisenmenger, W. Surf. Sci. 1998, $418,91$.

(22) Fang, H. B.; Giancarlo, L. C.; Flynn, G. W. J. Phys. Chem. B 1998, 102,7311

(23) Giancarlo, L. C.; Fang, H. B.; Rubin, S. M.; Bront, A. A.; Flynn, G. W. J. Phys. Chem. B 1998, 102, 10255.

(24) Edelwirth, M.; Freund, J.; Sowerby, S. J.; Heckl, W. M. Surf. Sci. 1998, 417, 201 297.

(25) Giancarlo, L. C.; Flynn, G. W. Annu. Rev. Phys. Chem. 1998, 49,

(26) Stevens, F.; Beebe, T. P. Langmuir 1999, 15, 6884.

(27) Giancarlo, L. C.; Fang, H.; Avila, L.; Fine, L. W.; Flynn, G. W. J. Chem. Educ. 2000, 77, 66.

(28) Furukawa, M.; Tanaka, H.; Kawai, T. Surf. Sci. 2000, 445, 1.

(29) Giancarlo, L. C.; Flynn, G. W. Acc. Chem. Res. 2000, 33, 491.

(30) Gesquiere, A.; Abdel-Mottaleb, M. M.; De Feyter, S.; De Schryver, F. C.; Sieffert, M.; Mullen, K.; Calderone, A.; Lazzaroni, R.; Bredas, J. L. Chem.-Eur. J. 2000, 6, 3739.

(31) Yablon, D. G.; Giancarlo, L. C.; Flynn, G. W. J. Phys. Chem. B 2000, 104, 7627 .

(32) Yablon, D. G.; Guo, J. S.; Knapp, D.; Fang, H. B.; Flynn, G. W. J. Phys. Chem. B 2001, 105, 4313.

(33) Yablon, D. G.; Wintgens, D.; Flynn, G. W. J. Phys. Chem. B 2002, 106,5470 .

(34) Han, M.-J.; Xu, Q. M.; Wan, L. J.; Bai, C. L. J. Phys. Chem. B 2002, 106, 11272
(35) Ishikawa, Y.; Ohira, A.; Sakata, M.; Hirayama, C.; Kunitake, M. Chem. Commun. 2002, 22, 2652.

(36) Samori, P.; Severin, N.; Simpson, C. D.; Mullen, K.; Rabe, J. P. J. Am. Chem. Soc. 2002, 124, 9454.

(37) Samori, P.; Simpson, C. D.; Mullen, M.; Rabe, J. P. Langmuir 2002, $18,4183$.

(38) Samori, P.; Rabe, J. P. J. Phys.: Condens. Matter 2002, 14, 9955 (39) Lackinger, M.; Griessl, S.; Heckl, W. M.; Hietschold, M. Anal. Bioanal. Chem. 2002, 374, 685.

(40) Chen, Q.; Frankel, D. J.; Richardson, N. V. Langmuir 2002, 18 , 3219 .

(41) Barth, J. V.; Weckesser, J.; Trimarchi, G.; Vladimirova, A. D. V.; Cai, C.; Brune, H.; Gunter, P.; Kern, K. J. Am. Chem. Soc. 2002, 124, 7991.

(42) Miura, A.; Chen, Z. J.; Uji-i, H.; De Feyter, S.; Zdanowska, M.; Jonkheijm, P.; Schenning, A.; Meijer, E. W.; Wurthner, F.; De Schryver, F. C. J. Am. Chem. Soc. 2003, 125, 14968.

(43) Theobald, J. A.; Oxtoby, N. S.; Phillips, M. A.; Champness, N. R.; Beton, P. H. Nature 2003, 424, 1029.

(44) Li, C. J.; Zeng, Q. D.; Wan, L. J.; Xu, S. L.; Wang, C. R.; Bai, C. L. J. Phys. Chem. B 2003, 107, 747 .

(45) Wintgens, D.; Yablon, D. G.; Flynn, G. W. J. Phys. Chem. B 2003 107, 173.

(46) De Feyter, S.; Gesquière, A.; Klapper, M.; Mullen, K.; De Schryver, F. C. Nano Lett. 2003, 3, 1485.

(47) Samori, P.; Yin, X. M.; Tchebotareva, N.; Wang, Z. H.; Pakula, T.; Jackel, F.; Watson, M. D.; Venturini, A.; Mullen, K.; Rabe, J. P. J. Am. Chem. Soc. 2004, 126, 3567.

(48) Watson, M. D.; Jackel, F.; Severin, N.; Rabe, J. P.; Mullen, K. J. Am. Chem. Soc. 2004, 126, 1402.

(49) Wei, Y.; Kannappan, K.; Flynn, G. W.; Zimmt, M. B. J. Am. Chem. Soc. 2004, 126, 5318 .

(50) Lackinger, M.; Müller, T.; Gopakumar, T. G.; Müller, F.; Hietschold, M.; Flynn, G. W. J. Phys. Chem. B 2004, 108, 2279.

(51) Müller, T.; Werblowsky, T. L.; Florio, G. M.; Berne, B. J.; Flynn, G. W. Proc. Natl. Acad. Sci. U.S.A. 2005, 102, 5315.

(52) De Feyter, S.; De Schryver, F. C. J. Phys. Chem. B 2005, 109, 4290.

(53) Florio, G. M.; Werblowsky, T. L.; Muller, T.; Berne, B. J.; Flynn, G. W. J. Phys. Chem. B 2005, 109, 4520.

(54) De Feyter, S.; Miura, A.; Yao, S.; Chen, Z.; Wurthner, F.; Jonkheijm, P.; Schenning, A. P. H. J.; Meijer, E. W.; De Schryver, F. C. Nano Lett. 2005, 5, 77.

(55) Fukumara, H.; Daizo, L.-I.; Uji-I, H.; Nishio, S.; Sakai, H.; Ohuchi, A. ChemPhysChem 2005, 6, 2383.

(56) Kampschulte, L.; Lackinger, M.; Maier, A.-K.; Kishore, R. S. K.; Griess, S.; Schmittel, M.; Heckl, W. M. J. Phys. Chem. B 2006, 110, 10829.

(57) Mamdouth, W.; Dong, M.; Xu, S.; Rauls, E.; Besenbacher, F. J. Am. Chem. Soc. 2006, 128, 13305

(58) Barth, J. V. Angew. Chem., Int. Ed. 2000, 39, 1230.

(59) Beardmore, K. M.; Kress, J. D.; Bishop, A. R.; Grønbech-Jensen, N. Synth. Met. 1997, 84, 317 .

(60) Buehler, M. J. J. Comput. Theor. Nanosci. 2006, 3, 603.

(61) De Feyter, S.; Miura, A.; Yao, S.; Chen, Z.; Wurthner, F.; Jonkheijm, P.; Schenning, A. P. H. J.; Meijer, E. W.; De Shryver, F. C. D. Nano Lett. 2005, 5, 77.

(62) Feldheim, D. L.; Keating, C. D. Chem. Soc. Rev. 1998, 27, 1.

(63) Goetz, R.; Lipowsky, R. J. Chem. Phys. 1998, 108, 7397.

(64) Heimel, G.; Romaner, L.; Brédas, J.-L.; Zojer, E. Surf. Sci. 2006 $600,4548$.

(65) Judkins, C. M.; Bohannan, E. W.; Herbig, A. K.; Powers, J. A.; Van Galen, D. A. J. Electroanal. Chem. 1998, 451, 39.

(66) Liu, J.; Kim, A. Y.; Wang, L. Q.; Palmer, B. J.; Chen, Y. L.; Bruinsma, P.; Bunker, B. C.; Exarhos, G. J.; Graff, G. L.; Rieke, P. C.; et al. Adv. Colloid Interface Sci. 1996, 69, 131.

(67) Mayer, B.; Klein, C. T.; Topchieva, I. N.; Köhler, G. J. Comput.Aided Mol. Des. 1999, 13, 373.

(68) Pidaparti, R. M.; Murugesan, K.; Yokota, H. J. Comput. Theor Nanosci. 2006, 3, 643.

(69) Plass, K. E.; Kim, K.; Matzger, A. J. J. Am. Chem. Soc. 2004, 126,9042

(70) Segev, L.; Salomon, A.; Natan, A.; Cahen, D.; Kronik, L.; Amy, F.; Chan, C. K.; Kahn, A. Phys. Rev. B 2006, 74, 165323.

(71) Sowerby, S. J.; Edelwirth, M.; Heckl, W. M. J. Phys. Chem. B 1998, 102, 5914 .

(72) Hansen, F. Y.; Herwig, K. W.; Matthies, B.; Taub, H. Phys. Rev. Lett. 1999, 83, 2362.

(73) Yaliraki, S. N.; Longo, G.; Gale, E.; Szleifer, I.; Ratner, M. A. J. Chem. Phys. 2006, 125, 074708 .

(74) Hentschke, R.; Schurmann, B. L.; Rabe, J. P. J. Chem. Phys. 1992, 96, 6213.

(75) Keeling, D. L. Nano Lett. 2003, 3, 9. 
(76) Lehn, J.-M. Supramolecular Chemistry; VCH: Weinheim, Germany, 1995.

(77) Tabellion, F. M.; Seudel, S. R.; Arif, A. M.; Stang, P. J. J. Am. Chem. Soc. 2001, 123, 7740 .

(78) Yokoyama, T.; Yokoyama, S.; Kamikado, T.; Okuno, Y.; Mashiko, S. Nature 2001, 413, 619 .

(79) Whitesides, G. M.; Simanek, E. E.; Mathias, J. P.; Seto, C. T.;

Chin, D. N.; Mammen, M.; Gordon, D. M. Acc. Chem. Res. 1995, 28, 37.

(80) Seto, C. T.; Whitesides, G. M. J. Am. Chem. Soc. 1990, 112, 6409.

(81) Ray, P. C.; Das, P. K. Chem. Phys. Lett. 1997, 281, 243.

(82) Ranganathan, A.; Pedireddi, V. R.; Rao, C. N. R. J. Am. Chem. Soc. 1999, 121, 1752

(83) Damodaran, K.; Sanjayan, G. J.; Rajamohanan, P. R.; Ganapathy, S.; Ganesh, K. N. Org. Lett. 2001, 3, 1921.

(84) Pedireddi, V. R.; Belhekar, D. Tetrahedron 2002, 58, 2937.

(85) Data from NIST Standard Reference Database 69 - March Release: NIST Chemistry WebBook.

(86) Liang, X.; Zheng, W.; Wong, N.-B.; Li, J.; Tian, A. THEOCHEM 2004, 672, 151 .

(87) Klotz, I. M.; Askounis, T. J. Am. Chem. Soc. 1947, 69, 801.

(88) Newman, R.; Badger, R. M. J. Am. Chem. Soc. 1952, 74, 3545.

(89) Pedireddi, V. R.; Chatterjee, S.; Ranganathan, A.; Rao, C. N. R. J. Am. Chem. Soc. 1997, 119, 10867.
(90) Coppens, P.; Vos, A. Acta Crystallogr., Sect. B 1971, 27, 146.

(91) Verschoor, G. C.; Keulen, E. Acta Crystallogr., Sect. B 1971, 27 , 134.

(92) Ranganthan, A.; Pedireddi, V. R.; Sanjayan, G.; Ganesh, K. N.; Rao, C. N. R. J. Mol. Struct. 2000, 522, 87.

(93) Stern, H. A.; et al. SIM molecular dynamics simulation program. (94) Jorgensen, W. L.; Maxwell, D. S.; Tirado-Rives, J. J. Am. Chem. Soc. 1996, 118, 11225 .

(95) JAGUAR; Schrödinger, Inc.: Portland, OR, 2000

(96) Schlick, T.; Fogelson, A. ACMT Math Software 1992, 18.

(97) Schlick, T.; Fogelson, A. A Powerful Truncated Newton Method for Potential Energy Minimization. J. Comput. Chem. 1987, 8, 1025.

(98) Martyna, G. J.; Klein, M. L.; Tuckerman, M. J. Chem. Phys. 1992, 97, 2635.

(99) Nose, S. J. Chem. Phys. 1984, 81, 511.

(100) Steele, W. A. Surf. Sci. 1973, 36, 317

(101) Steele, W. A.; Vernov, A. V.; Tildesley, D. J. Carbon 1987, 25,

(102) Jorgensen, W. L. J. Phys. Chem. 1986, 90, 1276.

(103) Griessl, S.; Lackinger, M.; Edelwirth, M.; Hietschold, M.; Heckl,

W. M. Single Mol. 2002, 3, 25.

(104) Unpublished results. 